\title{
Performance Evaluation Of Grid-Based ROUTING PROTOCOL FOR UNDERWATER WIRELESS SENSOR NETWORKS UNDER DIFFERENT MOBILITY MODELS
}

\author{
Z. Alkindi, N. Alzeidi, B. Arafehand A.Touzene \\ Department of Computer Science, Sultan Qaboos University, Muscat, Oman
}

\begin{abstract}
In this paper, we have conducted a simulation study to evaluate Multipath Grid-Based Geographic Routing Protocol (MGGR) under different selected mobility models. The protocol has been evaluated under three mobility models (i.e. Random Way Point, Reference Point Group and Meandering Current Mobility Model) using Aqua-Sim NS2-based simulator. An Extensive number of experiments have been conducted to assess the packet delivery ratio, the end-to-end-delay and power consumption under different operating conditions. We have noticed that the performance of MGGR is only marginally affected by the mobility behavior of nodes especially with the condition where the speed of nodes movements increased. Nevertheless, the evaluation has also shown that MGGR exhibit only average performance when used in coastal areas. This is evident as the MCMM (used primarily to model the movement of nodes in coastal areas) gave the lowest performance compared to other models.
\end{abstract}

\section{KEYWORDS}

UWSNs, Mobility models, Routing Protocols, Aquatic communication, Simulation, and MGGR

\section{INTRODUCTION}

Underwater wireless sensor networks (UWSNs) have attracted a growing research interest from the wireless networks community. A number of applications are envisioned to benefit from these networks including security and military surveillance, oceanography data collection, ecology, disaster prevention, and offshore exploration [1]. Most of the research studies rely on simulation experiments to effectively evaluate the performance of different techniques and to explore the design space of UWSN. UWASNs consist of sensor nodes deployed in a specific oceanic underwater area and use acoustic signals for communication. These collaborative sensor nodes are used to perform various monitoring tasks e.g. offshore exploration, intrusion detection and target tracking, guided navigation, and public safety (e.g. disaster prevention, seismic and tsunami monitoring) [2] [3]. Researchers who study UWSNs have addressed a number of issues, for instance synchronization [4], data gathering [5], localization, routing and mobility. Node mobility is one of the main concerns that should be considered during the implementation of WSN generally and UWSN particularly. This is due to numerous factors in underwater environment, such as water currents and underwater living creatures, which lead to dispersion of sensor nodes [6]. Mobility models are used in simulation experiments to capture, with some level of accuracy, the movement behavior of nodes. To successfully conduct these studies, it is crucial to use mobility models that closely mimic the mobility behavior of sensor nodes. Several mobility models are used for simulation experiments in terrestrial wireless sensor networks including a random walk and a random way point model. The effects of these mobility models on the performance of routing protocols have been investigated by a number of researchers. Conclusions

DOI: $10.5121 /$ ijwmn.2018.10102 
of these studies can't be generalized to UWSNs not only due to the different environment and used communication medium but also to the difficulty and inaccuracy of obtaining localization information. To the best of our knowledge, there have been no attempts to study the effects of mobility models on the performance of UWSNs routing protocols. Therefore, this paper presents the first performance evaluation of a grid-based routing protocol under different mobility models. MGGR is a grid-based routing protocol MGGR which has been recently proposed [7]. Evaluation of the MGGR under Random Way Point [8] mobility model has demonstrated its advantage against its counterparts. Therefore, the aim of this paper is to conduct a simulation-based comparative study to evaluate the performance of MGGR for UWSN under several mobility models. The rest of the paper is organized as follows. In section 2, related studies are presented, then, a description of selected mobility models and the MGGR are highlighted. Section 3 is dedicated to the performance analysis of the MGGR under some selected mobility models. Section 4 concludes the paper and highlights some directions for future work.

\section{RELATED WORK}

A mobility model is used to represent the node's movement pattern where different features of the nodes e.g. speed, direction, distance and node movement are well described [9]. If a node is steady in a particular position for a period of time, this time is called pause time [10]. In various network environments including TWSNs and UWSNs, several factors affect sensor nodes. In TWSNs nodes are usually static; the movement of these sensors is less than its movement in the underwater environment. In underwater, the water currents, wind, water temperature and underwater creatures influence the movement of the nodes which lead to major challenges for underwater localization and mobility modeling [11].

Generally, mobility models can be classified based on different criteria e.g. measurement, the scale of mobility, randomness, geological constraints, and destination oriented. There are two types of mobility models, trace based mobility models and synthetic mobility models. Trace mobility models are based on the realistic movement patterns of the nodes and their statistical properties. However, traces are typically limited in terms of availability and limited to specific scenarios. Synthetic mobility models, on the other hand, are models which have been established based on the analytical analysis of specific scenarios [9] [10]. Trace-based models include model$\mathrm{T}$, Manhattan and a simple human mobility model [4]. There are also hybrid mobility models like city section model and weighted waypoint model [9] [10].

Based on the type of movements, mobility models can be further classified as individual mobility models and group mobility models. In individual mobility models, nodes move independently of each other. Examples of synthetic-based individual mobility models include random walk, probabilistic random, random waypoint, random direction and gauss-Markov [4]. Group mobility models, on the other hand, model the movement behavior for groups of nodes, which move together to a common target location. One of these models is Reference Point Group Mobility Model which is classified by [10] as a synthetic-based group mobility model. In the following subsections, we elaborate more on some selected mobility models.

\subsection{SELECTEd Mobility ModelS}

\subsubsection{Random Waypoint (RWP)}

This is a random probability distribution model which is most commonly used to simulate the movement actions of a mobile network node in a given area[12][13]. In this model, a node randomly chooses a target point, which is called waypoint, in the area and start moving with constant speed on a straight line to the target waypoint (i.e. destination). After it waits at that 
position for a certain amount of pause time, a node chooses a new destination then moves with constant speed to the new waypoint, and so on. The node movement from a starting position to its end is represented as one movement period or transition. The above procedure of picking a random target position and random speed to move continues till reaching a predefined simulation time [9]. To determine the mobility behavior of a node, the model should consider a node's speed, a node's direction and a node's acceleration. The random waypoint model is developed to mimic the movement pattern of mobile nodes in a simplified implementation and analysis manner. Therefore, it may not effectively simulate some of realistic mobility characteristics e.g. temporal dependency, spatial dependency and geographic restriction [14].

\subsubsection{Reference Point Group Mobility Model (RPGM)}

In [15], the authors defined RPGM as simulation model which captures group mobility behavior of the mobile nodes. [10] Stated that RPGM is the primary group mobility model and mostly applied in various scenarios e.g. military battlefield communications and firemen rescue team. In each mobility group, the model states a reference midpoint. This center point known as logical center or team leader, determines the movement pattern for all nodes in the group [16] [10]. For instance, $(\mathrm{x}, \mathrm{y})$ is a physical point of the group's reference midpoint and its sensor node members are given by two levels of displacement vectors which are group motion vector and nodedependent random motion vectors. The group motion vector maps out the position of the reference point, while the node-dependent random motion vectors, added to the group motion vector, gives the locations of the nodes [15]. So, each node has its own space, speed and direction where the node exhibits random movement around the reference point, then the different nodes use their own mobility model and are added to the center point which guide the nodes to follow the direction of the group [17]. The authors in [16] [17] described the overall motion and movement of the group leader and the node members. The reference point will be moving based on a time denoted by $\mathrm{t}$ which can be represented by a motion vector $V_{\text {group }}^{t}$. Motion vector $V_{\text {group }}^{t}$ can be randomly selected or calculated based on certain predefined paths. A path which a group will follow is given by defining a sequence of check points along the path equivalent to given time intervals. Moreover, each node is allocated with a reference point that follows the group movement and per this center point, each node could be randomly located in the neighborhood. The motion vector of group member, i, at time $\mathrm{t}, V_{i}^{t}$, can be calculated as:

$$
V_{i}^{t}=V_{\text {group }}^{t}+R M_{i}^{t}
$$

Where the motion vector $R M_{i}^{t}$ is, a random vector deviated by group member i from its individual reference point. The vector $R M_{i}^{t}$ is an independent distributed random process whose length is equivalently distributed in the interval of $[0, \square \max ]$ (where $\square \max$ is maximum allowed distance deviation) and whose direction is uniformly distributed in the interval [0,2 $\square$ ]. The vector RMi determine how much the movement of group members deviate from their leader [17].

\subsubsection{Meandering Current Mobility Model (MCMM)}

In [3], Caruso et al. introduced a mobility model that is designed for underwater environment, named the Meandering Current Mobility Model (MCMM). This mobility model is a representative for coastal areas. In this model, sensor nodes are subjected to move by the effect of meandering sub-surface currents and vortices [2]. Consistent with the model movement scenario, the initial deployment of nodes will be done in a small subarea and then another batch of sensor nodes can be deploying to the same area and so on. In each deployment of nodes, the nodes will move under the effect of a meandering current. Therefore, the node's mobility in this model is confined to a certain plane. The MCMM is based on stream-function that have been proposed by [18], this function is designed to catch the two key features of a typical ocean flow which are 
currents and vortices. The non-dimensional form of the stream-function that describe the meandering jet movement is denoted by the following equation:

$$
\psi(x, y, t)=1-\tanh \left(\frac{y-B(t) \sin (k(x-c t))}{\sqrt{1+k^{2} B^{2}(t) \cos ^{2}(k(x-c t))}}\right)
$$

where $\mathrm{B}(\mathrm{t})=\mathrm{A}+€ \cos (\omega \mathrm{t})$, the parameter $\mathrm{k}$ represents the number of meanders in the unit length, $c$ is the phase speed with which they shift downstream. The time-dependent function $\mathrm{B}$ determines the width of the meanders, A modulates the average meander width, $€$ is the amplitude of the modulation, which has a peak speed inside the jet, and $\omega$ sets is its frequency $[3][20]$.

\subsection{Evaluation Of Routing Protocols Under Different Mobility Models}

It is commonly understood that mobility of nodes will have a significant influence on the performance of the routing protocols in different mobile wireless networks. The authors in [21] studied the performance of four main routing protocols which are Dynamic Source Routing (DSR), Location Aided Routing(LAR), Optimized Link State Routing(OLSR) and Zone Routing Protocol(ZRP) under different Mobility Models. The researchers changed the node density and speed during the evaluation of routing protocols with RWP, Gauss Markov (GM), Reference Point Group Mobility (RPGM) and Manhattan Grid (MG). The paper illustrated that LAR and OLSR show better performance with the variation of speed, node density and mobility pattern [21]. The authors used Bonn Motion [24] to generate the mobility models. The evaluation was done with varying the number of nodes from 5 to 200 and the speed between 0 to $20 \mathrm{~m} / \mathrm{s}$. The overall throughput showed that LAR and OLSR perform better under RPGM than the other mobility models.

F. Bai et al. [22] proposed a framework named IMPORTANT which was used to assess the impact of mobility on performance of routing protocols for ad hoc networks. The mobility models used were RWP, RPGM, Freeway mobility and Manhattan mobility model. The number of nodes was fixed at 40. NS 2 was used as a simulation tool. The authors evaluated three main routing protocols DSR, AODV and Destination-Sequenced Distance-Vector Routing (DSDV) under the above mobility models. They showed that performance of each protocol shows drastic differences across mobility models.

In [23], Atsan et. al. provided a survey and a classification of existing mobility models, then compared the performance of mobility models for MANET protocols. The authors selected AODV as the routing protocol and they used SWANS as network simulator. Four main mobility models were considered including random direction, boundless simulation area model, random walk and random waypoint model. The performance of the routing protocol has been tested using various metrics e.g. average message activity, average route request, completion rate and average RREQ messages sent per added route. Authors concluded that RWP is the most affected model from the increase in network density However, Direction and Random Walk mobility models showed the worst performances for AODV routing in simulations [23].

Despite the studies of the effect of mobility models on the performance of routing protocols in terrestrial networks, to the best of our knowledge, this effect has not been investigated for the underwater environment. Therefore, in this paper we present the first performance evaluation of a grid-based routing protocol (i.e. MGGR [8]) under three different mobility models which are RPGM, RWP and MCMM. 


\subsection{Multipath Grid-Based Geographic Routing Protocol For Uwsns (MGGR)}

MGGR is a grid-based routing protocol designed for underwater wireless sensor networks. The protocol forwards packets in a cell-by-cell fashion using available disjoint paths from a source node to the target node. Each grid cell is perceived as a cube and all grid cells have equal size denoted by $d 3$, where $d$ is the length of the cell side as shown in Figure 1 [7]. The Grid cells are identified by their 3D coordinates ( $\mathrm{x}, \mathrm{y}$ and $\mathrm{z}$ ). All sensor nodes in underwater environment communicate via acoustic signals thus, MGGR assumes that sensor nodes have the same features and capabilities e.g. transmission range, storage space and initial energy level. Moreover, these sensor nodes are deployed at different depths and are mobile. Each node can move with water currents as they are either attached to surface buoys, or they are anchored to the bottom of the ocean. In addition, there is a unique ID for every sensor node (NID) and grid coordinates id (GID). The length of the cell side (d) is calculated based on the transmission range $\mathrm{R}$ of the sensor nodes. It is chosen so that a node in a cell is capable to communicate with any node in its 26 neighboring cells. It is also assumed that a sensor node can gain location information for itself, for the neighboring sensor nodes and for the sink node. The authors [7] assumed that there is only one sink node which is fixed at the center of the top surface of the grid. MGGR depends on a gateway election algorithm. Generally, gateways are those nodes, which are responsible to forward data packets to the destination node. The gateways' information gets updated using a minimum set of control packets. When a gateway node has a packet to forward, it loops over a set of pre-computed cell disjoint paths in a round-robin fashion. If an empty cell is encountered (i.e. no node to forward the packet), MGGR provides a negative acknowledgment mechanism which sends a notification to previous gateway. Upon receiving a negative acknowledgement, the gateway marks the path with empty cell as a faulty path and attempts transmitting the data packet using another path from the set of alternative disjoint paths [7][8]. Evaluation study has shown that MGGR outperforms its counterparts in terms of power consumption, packet delivery ratio and end-to-end delay.

\section{Performance Evaluation}

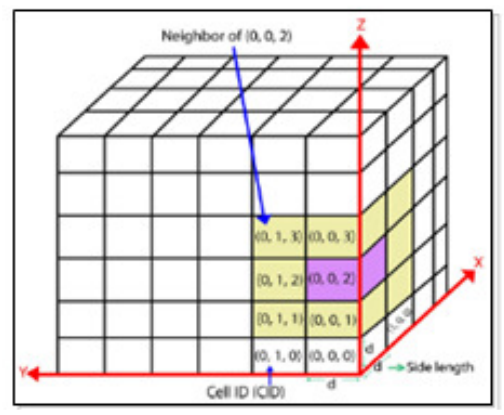

Figure 1: A view of 3D logical grid [7].

\subsection{EXPERIMENTAL SETUP}

The Abstract section begins with the word, "Abstract" in 13 pt. Times New Roman, bold italics, "Small Caps" font with a 6pt. spacing following. The abstract must not exceed 150 words in length in 10 pt. Times New Roman italics. The text must be fully justified, with a 12 pt. paragraph spacing following the last line. 
The performance of MGGR under the selected mobility models described in section two has been evaluated using the Aqua-Sim simulator. Aqua-Sim is based on NS-2, which is one of the most widely used network simulators. Moreover, to use the mobility models with MGGR, we have used enhanced Bonn Motion tool where the mobility models produced based on the requirements that fit with MGGR Tcl. Bonn-Motion is an open source Java software which is used to generate and analyze different mobility scenarios and is most commonly used as a tool for the investigation of mobile ad hoc network characteristics [24]. As mentioned in section two, Meandering Current Mobility Model (MCMM) is based on stream-function. Due to unavailability of this model in Bonn-Motion, we have developed the full implementation setup for MCMM. Like RPGM and RWP, the sink node has been initialized from MGGR Tcl and the scenario file designed to contain the node coordinates and speed. The following parameters determined the stream-function that followed by the MCMM to describe the node movement:

$$
\mathrm{A}=1.2, \mathrm{c}=0.12, \mathrm{k}=2.0 * \mathrm{M} \_\mathrm{PI} / 7.5,0.4, \text { epsilon }=0.3 \text {, }
$$

Where $\mathrm{A}$ is the average meander width, c defines the phase speed, epsilon indicates the amplitude of the modulation which has a peak speed inside the jet, and $\mathrm{k}$ states its frequency [3].

Nodes are deployed at different depths and can exhibit different types of movements either due to the currents or because of some sort of synthetic movement. However, oceans are a stratified, rotating fluid, hence vertical movements are, almost everywhere, negligible with respect to the horizontal ones [3]. Thus, we will assume that the sensors nodes move on horizontal surfaces, and neglect their vertical displacements. There is only one sink node and it is fixed at the middle of the top surface of the grid. So, this sink node can communicate using acoustic channels with the various underwater sensor nodes while it uses radio channels to interconnect with other sink nodes or terrestrial stations.

The following performance metrics have been used to evaluate the performance of MGGR under the three mobility models.

1. Packet Delivery Ratio (PDR): PDR as explained by [8] [9] [17] is the ratio of total number of packets that have been successfully sent by the source nodes and received by the destination nodes. It is calculated by dividing the number of packets received by target node over the number of packets sent from the source node.

$$
P D R=\sum \frac{\text { Number of packets received }}{\text { Number of packets send }}
$$

2. Average End to End Delay: defined by [9] [8] as the average delay time that is taken by a data packet to be sent from sender node to receiver node. This delay can be calculated as

$$
\mathrm{AED}=\sum_{i=0}^{n} \frac{\left(\text { time packet received }_{i}-\text { time packet sent }_{i}\right)}{\text { total number of packets received }}
$$

3. Energy Consumption: overall energy that is used during the simulation process. It contains the transmission power, the reception energy and the idling power spent by all nodes[8]:

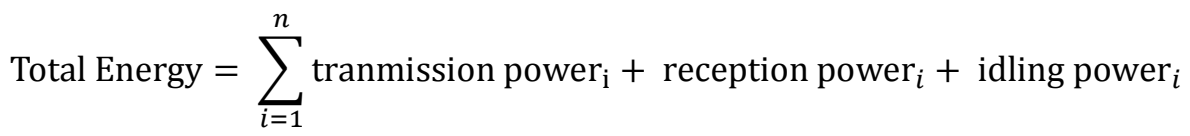


The effects of mobility models on MGGR have been tested under three main system parameters. These are: network density, traffic load and node mobility. Network density can be evaluated by changing the number of nodes in the network. The effect of traffic load has been evaluated by subjecting the network to different packet generation rates. Finally, the node maximum speed has been used to assess the effect of node mobility in the performance of the protocol. Table 1 lists a more comprehensive set of simulation parameters that have been used in the evaluation of the performance of MGGR along with different mobility models. The values of most of the general parameters have been taken from the literature [7].

Table 1 General System parameters.

\begin{tabular}{|l|l|}
\hline Parameter & Value(s) \\
\hline Simulator & Aqua-Sim \\
\hline Routing protocols & MGGR \\
\hline Topology size & $\left(3000^{*} 3000^{*} 3000\right) \mathrm{m}^{3}$ \\
\hline Channel & Underwater channel \\
\hline Propagation & Underwater propagation \\
\hline Initial energy & $10000 \mathrm{Joule}$ \\
\hline Transmission range (R) & $1500 \mathrm{~m}$ \\
\hline Simulation time & $1000 \mathrm{sec}$. \\
\hline Packet size & 200 bytes \\
\hline Number of sources & 20 \\
\hline Mobility model & RWP, RPGM and MCMM \\
\hline Nodes position update interval & $0.3 \mathrm{sec}$. \\
\hline
\end{tabular}

\subsection{ReSUlts AND Discussions}

\subsubsection{Effects of network density}

The performance of the MGGR protocol has been assessed under different mobility models, namely: RWP, RPGM and MCMM. As depicted in Figure 2, the performance of PDR improves as the network density increases with various selected mobility models; this is predictable as the probability that each cell in the grid contains a gateway increases and the possibility of path breakage reduces. Hence data packets can be effectively delivered to the target sensors. Figure 3 show that the average end-to-end delay increases as the network density increases. However, when sensor nodes are subjected to RPGM mobility model, a slight decline can be noticed. The energy consumption grows linearly as the number of nodes increases as can be seen from Figure 4. In all cases, MGGR seems to benefit from the group mobility of nodes experienced by the RPGM. This might partially be due to the less randomness in node movements, which contributes to more stable routing paths.

\subsubsection{Effects of node mobility}

Figure 5 shows that the PDR of MGGR decreases as the node speed increases, we noticed that as the speed reaches $2 \mathrm{~m} / \mathrm{s}$, the difference between the PDR of the protocol MGGR under different mobility models gets close to zero which means that MGGR is not highly affected by the movement pattern of the nodes when the speed exceeds a threshold. Besides, there was a decrease in the delay as the node speeds increase as illustrated in Figure 6. We observed that the end-toend delay of the MGGR under RPGM mobility model exhibits the lowest delay compared to its counterparts. Finally, Figure 7 demonstrates that as the speed of nodes increases, the total power consumed in MGGR slightly descend. This might be due to dropping the packets earlier in the network as the void grid cells increase with node mobility. 
International Journal of Wireless \& Mobile Networks (IJWMN) Vol. 10, No. 1, February 2018

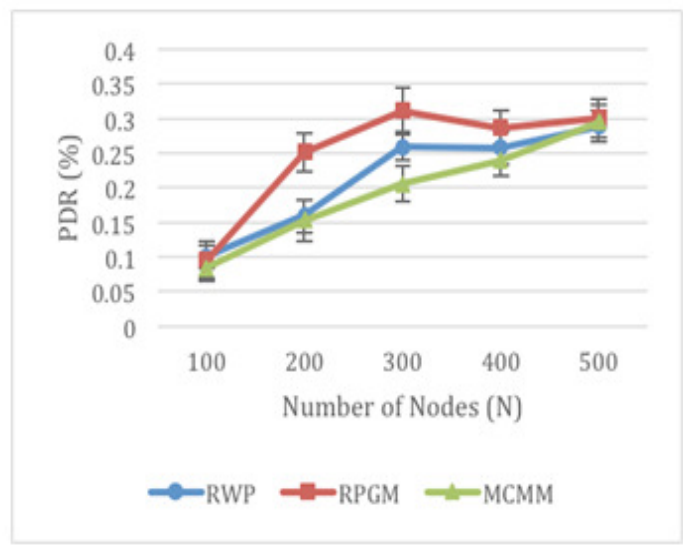

Figure 2: Effect of network density on PDR when using RWP, RPGM \& MCMM.

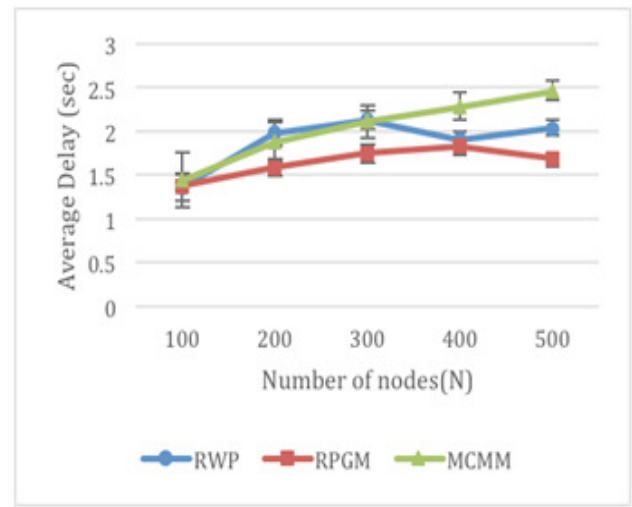

Figure 3: Effect of network density on the average end-to-end delay When using RWP, RPGM \& MCMM.

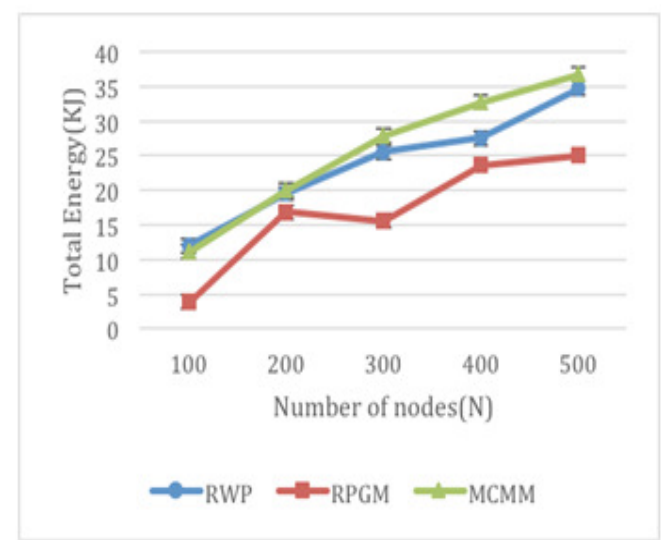

Figure 4: Effect of Network Density on Total Energy Consumption When using RWP, RPGM \& MCMM. 
International Journal of Wireless \& Mobile Networks (IJWMN) Vol. 10, No. 1, February 2018

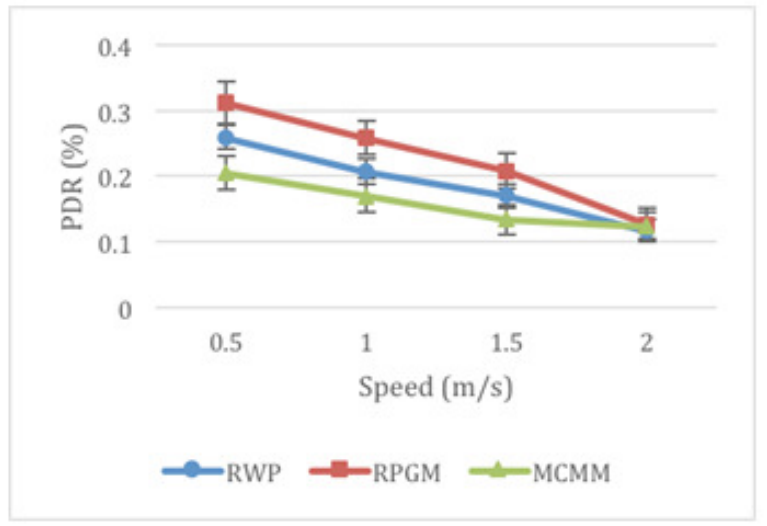

Figure 5: PDR Vs Speed When using RWP, RPGM \& MCMM.

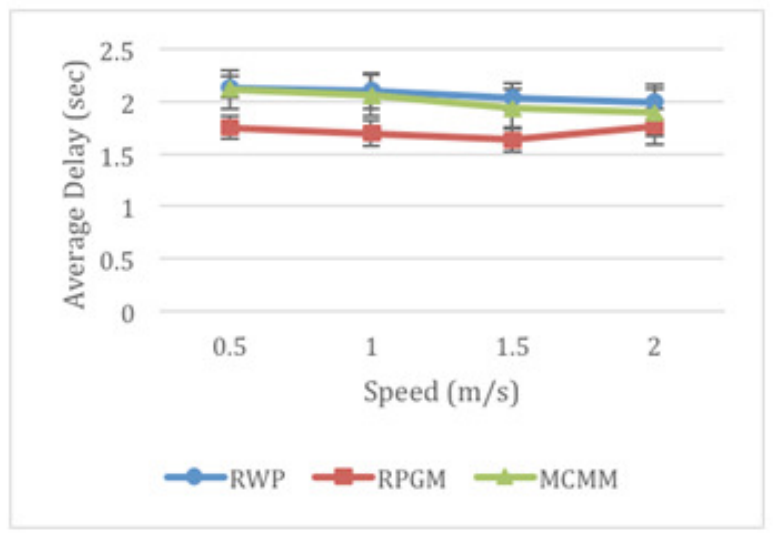

Figure 6: Average end-to-end delay vs Speed when using RWP, RPGM \& MCMM.

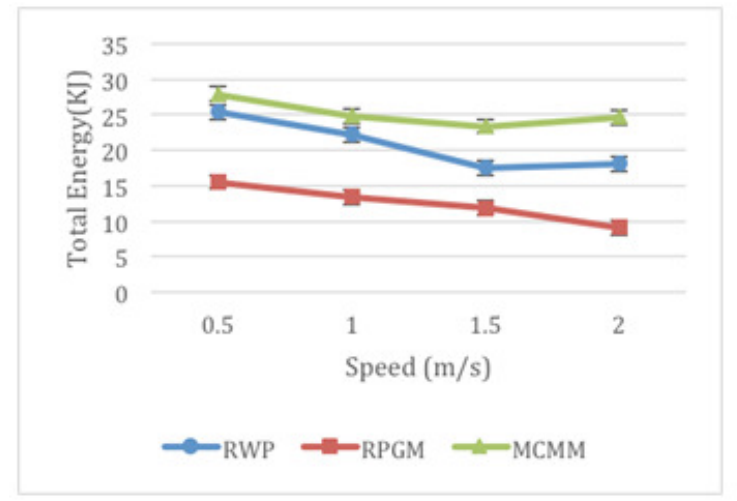

Figure 7: Total Energy Consumption Vs Speed when using RWP, RPGM \& MCMM.

\subsubsection{Effects of traffic load}

The impact of traffic load is evaluated by varying the mean of the exponential distribution of the packet generation rate from 0.025 to 0.15 packets per seconds. In this set of experiments, 300 nodes are deployed in the simulation area and the maximum node speed was set to $0.5 \mathrm{~m} / \mathrm{s}$. In Figure 8 the impact of traffic load on PDR is shown. As we can see that the PDR of all mobility 
models get decreased as the packet generation rate increased. The PDR while using the MCMM continues to be the lowest in all traffic loads. Nodes that are subject to MCMM mobility model tend to leave the simulation area and hence decrease the chance of packets being delivered to their destinations. RPGM, however, is achieving the best PDR as the nodes are scattered around a reference point and there is a less likelihood that they may leave their grid cells. Figure 9 shows the effect of traffic load on the average end-to-end delay. The figure demonstrates that there is a slight decrease in the average delay of the MGGR under all mobility models as the traffic load increases. This is mainly due to the packet drop rate as the traffic load increases and hence these dropped packets are not counted in the calculation of the end-to-end delay. It can also be noticed that as the traffic load increase, the protocol exhibits very similar end-to-end delays with various mobility models. Having said that, it can be still noticed that the end-to-end delay of MGGR under RPGM is lower than its counterparts. This is more apparent with low to medium traffic loads. Again, this can be explained by the level of randomness of nodes movements and hence longer path lifetimes. Figure 10, illustrates the increasing trend of the energy consumption as the traffic load increases. When we increased the traffic load, there is an increase in the packets sent and received and then the energy consumption gets increased. The energy consumed by MGGR with RPGM is again the lowest compared to the other two models due to the correlated movement of nodes, which may result in longer path lifetime.

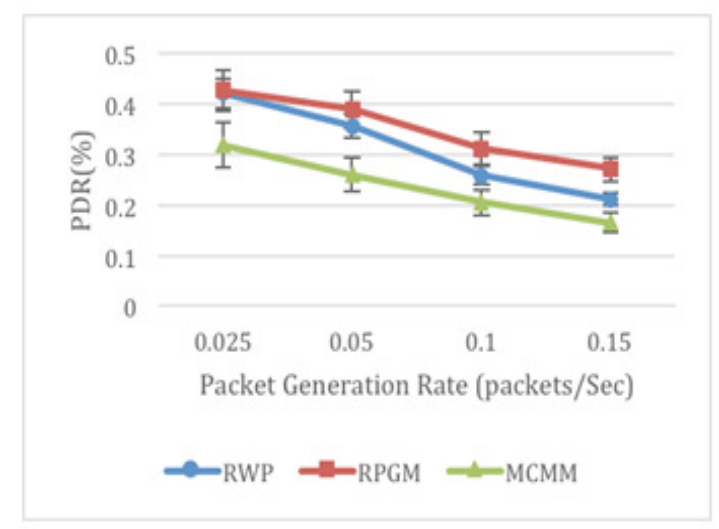

Figure 8: Effect of Traffic Load on PDR When using RWP, RPGM \& MCMM.

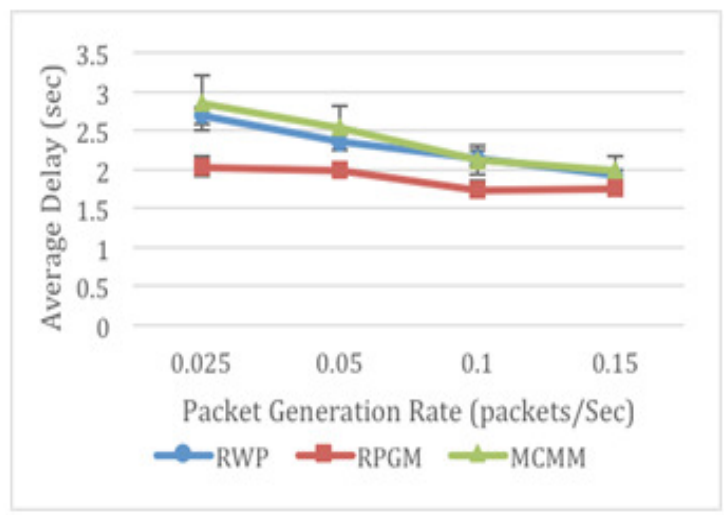

Figure 9 : Effect of Traffic Load on Average end-to-end delay When using RWP, RPGM \& MCMM. 


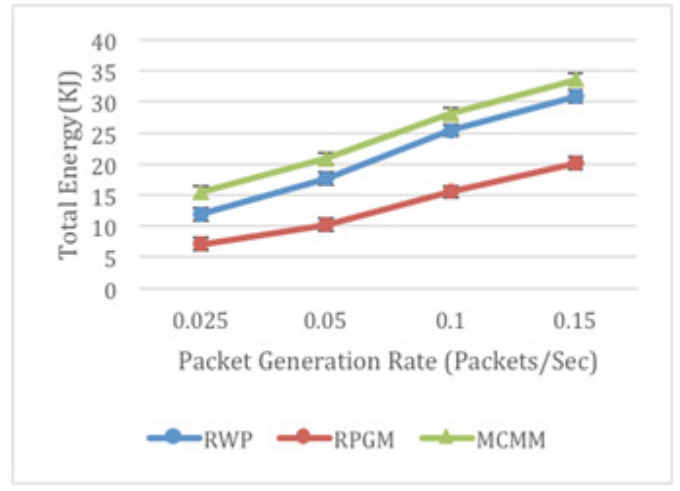

Figure 10: Effect of Traffic Load on Total Energy Consumption When using RWP, RPGM \& MCMM.

\section{Conclusions}

This paper presents the first performance analysis of a grid-based routing protocol (namely, MGGR) for UWSNs under different mobility models. The analysis has shown that MGGR is less sensitive to the mobility pattern of the sensor nodes. Moreover, it achieves good performance under different mobility models. This gives the protocol the advantage of being applicable to different application domains. However, the study has also shown that the group mobility of sensor nodes (illustrated by the RPGM) works in the favour of grid-based routing protocols due to the lower level of randomness exhibited by this type of movements. We plan to extend this work by experimenting with other mobility models and/or routing protocol to get a more comprehensive analysis. Moreover, the insights from this research can be used as a base to develop a mobility model designed specifically for the underwater environment.

\section{REFERENCES}

[1] S. Climent, A. Sanchez, J. V. Capella, N. Meratnia, and J. J. Serrano, "Underwater acoustic wireless sensor networks: advances and future trends in physical, MAC and routing layers.," Sensors (Basel)., vol. 14, no. 1, pp. 795-833, 2014.

[2] A. K. Mandal, S. Misra, T. Ojha, M. K. Dash, \& M. S. Obaidat, "Oceanic forces and their impact on the performance of mobile underwater acoustic sensor networks," International Journal of Communication Systems, 10.1002/dac.2882, 2014.

[3] F. Paparella, L. F. M. Vieira, M. Erol, and M. Gerla, "The Meandering Current Mobility Model and its Impact on Underwater Mobile Sensor Networks,” pp. 771-779, 2008.

[4] W. K. G. Seah, V. Chandrasekhar, and W. K. G. Seah, "Localization in underwater sensor networks Survey and challenges", In Proceedings of the 1st ACM international workshop on Underwater networks (pp. 33-40). ACM, 2006.

[5] A. Syed, W. Ye, B. Krishnamachari, and J. Heidemann, "Understanding Spatio-Temporal Uncertainty in Medium Access with ALOHA Protocols,". In Proceedings of the second workshop on Underwater networks (pp. 41-48). ACM, 2007.

[6] S. Climent, A. Sanchez, J. V Capella, J. J. Serrano, and U. Polit, "Study of MAC Protocols for a Real Underwater Sensor Network Application." In Proceedings of the International Conference on Wireless Networks (ICWN) (p. 1). The Steering Committee of The World Congress in Computer Science, Computer Engineering and Applied Computing (WorldComp), Las Vegas, NE, USA, 2012. 
International Journal of Wireless \& Mobile Networks (IJWMN) Vol. 10, No. 1, February 2018

[7] F. Al-salti, N. Alzeidi, and B. Arafeh, "A New Multipath Grid-Based Geographic Routing Protocol for Underwater Wireless Sensor Networks." In Cyber-Enabled Distributed Computing and Knowledge Discovery (CyberC), International Conference on IEEE, (pp. 331-336), 2014.

[8] F. Al Salti, N. Alzeidi, and B. R. Arafeh, "EMGGR: an energy-efficient multipath grid-based geographic routing protocol for underwater wireless sensor networks," Wireless Networks, pp.1-14, 2016.

[9] A. K. Shukla, "The Efficiency Analysis of Mobility Model using Routing Protocols," In International Conference on Advances in Computer Applications (ICACA), USA, pp. 6-10, 2012.

[10] A. Ribeiro and R. C. Sofia, “A Survey on Mobility Models for Wireless Networks,” SITI, University Lusófona, Tech. Rep. SITI-TR-11-01, 2011.

[11] Z. Zhou, S. Member, Z. Peng, and S. Member, "Scalable Localization with Mobility Prediction for Underwater Sensor Networks,” IEEE Transactions on Mobile Computing, 10(3), pp. 335-348, 2011.

[12] C. Bettstetter, H. Hartenstein, and X. Prez-Costa, "Stochastic properties of the random waypoint mobility model," Wireless Networks, 10(5), pp. 555-567, 2004.

[13] V. A. Davies, "Evaluating Mobility Models Within An Ad Hoc Network," Master's thesis, Colorado School of Mines, p.86, 2000.

[14] F. Bai and A. Helmy, "A Survey of Mobility Models in Wireless Adhoc Networks," WirelessAdhoc Networks, University of Southern California, USA, 206(147), pp. 1-30, 2004.

[15] K. H. Wang and B. Li, "Group mobility and partition prediction in wireless ad-hoc networks," In Communications, ICC 2002.IEEE International Conference on IEEE, 2, pp. 1017-1021, 2002.

[16] W. Romsaiyud, W. Premchaiswadi, and N. Premchaiswadi, "An Autonomous Group Mobility Prediction Model for Simulation of Mobile Ad-hoc through Wireless Network," J. Wirel.Netw. Commun., vol. 2, no. 5, pp. 126-135, 2012.

[17] G. Jayakumar and G. Ganapathi, "Reference Point Group Mobility and Random Waypoint Models in Performance Evaluation of MANET Routing Protocols,” J. Comput. Syst. Networks, Commun., vol. 2008, pp. 1-10, 2008.

[18] A. S. Bower, "A simple kinematic mechanism for mixing fluid parcels across a meandering jet," Journal of Physical Oceanography, 21(1), pp.173-180, 1991.

[20] Y. Ren, W. K. Seah\& P. D. Teal, "Performance of pressure routing in drifting 3D underwater sensor networks for deep water monitoring," In Proceedings of the seventh ACM international conference on underwater networks and systems, p. 28, ACM., 2012.

[21] D. Kumar and S. C. Gupta, "Routing Protocols under different Mobility Models , Node Density and Speed," Transactions on Networks and Communications, 3(1), pp.93, 2015.

[22] F. Bai, N. Sadagopan, A. Helmy, "The IMPORTANT Framework for Analyzing the Impact of Mobility on Performance of Routing for Ad Hoc Networks", AdHoc Networks Journal - Elsevier Science, 1(4), pp. 383-403, 2003.

[23] E. Atsan\& Ö. Özkasap, "A classification and performance comparison of mobility models for ad hoc networks," In International Conference on Ad-Hoc Networks and Wireless, pp. 444-457, Springer Berlin Heidelberg, 2006.

[24] M. Gerharz, C. de Waal, "Bonn Motion - a mobility scenario generation tool", University of Bonn, [Online]. Accessible: www.cs.uni-bonn.de/IV/BonnMotion/. 
International Journal of Wireless \& Mobile Networks (IJWMN) Vol. 10, No. 1, February 2018

[25] J. G. Proakis, E. M. Sozer, , J. A. Rice \& M. Stojanovic, "Shallow water acoustic networks," IEEE communications magazine, 39(11), PP.114-119, 2001.

[26] P. Noonsang, K. Jaroensutasinee, \& M . Jaroensutasinee, "Wireless Sensor Network for Ocean Observatory," Indian Journal of Geo-Marine Sciences, 44(7), pp. 941-950,2015. 